\title{
HIV and mental illness in Malawi and the neuropsychiatric sequelae of efavirenz
}

\author{
Andrew Drury1,2, Selena Gleadow-Ware ${ }^{2,3}$, Sheila Gilfillan ${ }^{2,4}$, Jen Ahrens ${ }^{2,5}$ \\ 1. South London and Maudsley NHS Foundation Trust \\ 2. Scotland Malawi Mental Health Education Project \\ 3. Honorary lecturer in psychiatry, University of Aberdeen \\ 4. Herdmanflat Hospital, NHS Lothian \\ 5. College of Medicine, Blantyre, Malawi
}

Date Received: 09 July 2017

Revision Received: 18 October 2017 Date Accepted: 05 November, 2017 Correspondence:

Dr. Andrew Drury

(drurya@doctors.org.uk)

https://dx.doi.org/10.4314/mmi.v30i1.9

Corresponding author: Andrew Drury; drurya@doctors.org.uk

Abstract
Introduction
Little is published about mental disorders in Malawi, specifically in relation to Human Immunodeficiency Virus (HIV) and it's
treatment. Efavirenz is a medication commonly used as part of triple therapy for HIV treatment. Indeed, in 2013, Malawi introduced
$5 \mathrm{~A}$ with Efavirenz as part of it's 1st line treatment for HIV. There exists some literature documenting known psychiatric side effects of
Efavirenz, which include anxiety, mood changes, nightmares, psychosis and suicidal ideation. Little is known about what features are
most common in the presentation and what factors in the patient and drug which may make this reaction more likely.
Aim
The aim of this commentary is to review the association between HIV and psychiatric disorder, and consider the neuropsychiatric
side-effects of Efavirenz.
Method
An evaluative literature review was completed by means of multiple electronic database search as well as an additional manual search
to obtain published works identified through the electronic search. Search terms used were: Efavirenz, Acquired Immunodeficiency
Syndrome, Africa, Antiretroviral Therapy, Developing Countries, Malawi, Mental Disorders, Public Health, and Psychiatry.
Conclusion
This is an important area of study, as potentially large numbers of individuals with HIV are being placed on Efavirenz as first line
treatment, yet $60 \%$ may experience some form of neuropsychiatric side effects.

\section{Introduction}

Acquired Immune Deficiency Syndrome (AIDS) is a disease caused by the Human Immunodeficiency Virus (HIV), as described by the Center for Disease Control in $1981^{1}$. More than 36 million people are currently living with HIV, the vast majority of whom are in low and middle-income countries $^{2}$. About 35 million people have already died of this disease $^{2}$. In 2012, an estimated 2.3 million people were newly infected with the virus and 1.1 million people died of AIDSrelated illnesses in 20152. According to the 2010 Malawi Demographic Health Survey (MDHS), the prevalence of HIV in adults aged 15 to 49 in Malawi is approximately $10-11 \%$, and the number of people living with HIV is 1.1 million ${ }^{3}$. The Joint United Nations Programme on HIV and AIDS (UNAIDS) recorded 36,000 new HIV infections and 24,000 AIDS-related deaths in 2016, although both of these figures represent a decrease from the 2010 figures $^{4}$.

There is a dearth of large-scale prevalence studies exploring mental illness in Malawi. Studies examining this have tended to be relatively small in scale and completed in the Southern region of Malawi. The prevalence of mental, neurological and substance use disorders for primary care attendees is usually between $15 \%$ and $30 \%$ and in some cases reaches as high as $45 \% 0^{5}$. Wright et al found that $28.8 \%$ of people attending primary care clinics have a common mental disorder including depression, ${ }^{6}$ and Stewart et al found a $30.4 \%$ prevalence of depressive episodes among women with young infants in Thyolo, as well as associations with poverty, relationship problems, HIV-infection and poor infant health ${ }^{7}$. Furthermore, the Malawi Health Sector Strategic Plan 20112016 indicates that mental illness accounts for 4\% of the total burden of disease in Malawi ${ }^{8}$.

Zomba Mental Hospital (ZMH) is the only governmentrun tertiary psychiatric referral hospital in Malawi. The commonest reasons for admission at ZMH are schizophrenia, bipolar disorders, intellectual disability, epilepsy, substancerelated and $\mathrm{HIV}$-related conditions ${ }^{9}$. Data from inpatients at Zomba suggests that $90 \%$ of patients admitted at ZMH are diagnosed with Schizophrenia or related illnesses ${ }^{10}$.

\section{Association between HIV and Psychiatric disorder}

$H I V$ infection in those with pre-existing mental disorder

It has been hypothesized that mental illness is a risk factor for developing HIV-infection. This is mainly based on indirect evidence ${ }^{11}$ and is thought to involve multiple factors, including; high-risk behaviours such as infrequent condom use, multiple sex partners and intravenous drug use, ${ }^{12,13}$ social exclusion that may lead to exchange of sex for money or goods, or forced sex, ${ }^{14,15}$ cognitive deficits leading to impaired judgment and/or the ability to negotiate safe $\operatorname{sex}^{16}$. In addition, the consequences of mental illness may lead to insufficient money to purchase condoms, no privacy for safer sex negotiation and considerable periods of hospitalization that may interfere with long-term sexual relationships and the opportunity to obtain condoms ${ }^{17}$. 
Depression has been identified as a major contributor to sexual risky behaviour resulting in HIV infection in Botswana and other countries ${ }^{18-24}$. It has been associated with steeper declines in CD4 counts, greater risk of developing HIV dementia, decreased antiretroviral medication adherence and more rapid progression to AIDS and death ${ }^{18}$. Manic symptoms such as increased energy, grandiosity, hyper sexuality, impulsivity, and poor judgment often lead to risky behaviours ${ }^{25-27}$. Over half of individuals with bipolar disorder also have lifetime substance use disorders, which further increase the risk of HIV infection ${ }^{25,27-28}$. Cognitive dysfunction in patients having primary psychiatric illness like schizophrenia has been well documented'. Cognitive deficits such as lack of planning, impaired judgement, lack of motivation to engage in safer sexual behaviours and inability to acquire and/or use information about HIV/ AIDS increase risk of contracting HIV 9 .

Studies from Africa, United States of America (USA) and elsewhere, investigating the link between mental illnesses and HIV, report varying but consistent results that adults with severe mental illness have higher rates of HIV infection ${ }^{29-34}$ Prevalence rates of HIV in those with mental illness range from $2-76$ times higher than the general population ${ }^{32,35-38}$.

There is less data available about the relationship between mental illness and the risk of contracting HIV in low-income countries. One systematic review found that the HIVprevalence varied between 0 and $23.8 \%$, which appeared to correspond to the prevalence in the general population ${ }^{39}$. The latter figure represented a study in Zimbabwe ${ }^{40}$. In Malawi, psychiatric outpatients in Thyolo had an HIV-prevalence of $14.8 \%$, compared to the general population of $21 \%{ }^{41}$.

Rates of mental disorder in those with pre-existing HIV

Mental illness and neuropsychiatric manifestations of HIV are often a combination of complex biological, psychological and social circumstances associated with HIV infection ${ }^{42}$. The following mechanisms have been hypothesized to be important: the psychological burden arising from the acute trauma of the diagnosis, difficulties posed on daily life, the longer-term threat of physical decline and shortened life expectancy ${ }^{11}$. Others are: the necessary life style changes, complicated therapeutic regimes, aversive symptoms and stigma leading to guilt and loss of social support, ${ }^{11}$ as well as the direct effects of the virus on the central nervous system causing neuropsychiatric complications, ${ }^{43}$ opportunistic infections of the nervous system, ${ }^{44}$ and side effects of antiretroviral therapy (ART) on the central nervous system ${ }^{45}$.

Documented information about mental health disorders among HIV-positive people in low and middle-income countries is less extensive. A systematic review from developing countries shows a wide range of prevalence of depression among people infected with HIV $(0-64 \%)^{15}$. This wide range is most likely representative of the wide variation in research methods applied. The review suggests that people infected with HIV are more often psychologically distressed and that this may be related to the severity of physical symptoms, the quality of family relations and the support of the partner ${ }^{15}$. This finding has been repeated in a Malawian setting ${ }^{46}$.

Depression has been documented among patients with HIV at $17.9 \%$ in Salima and 20\% among adolescents with HIV in Lilongwe ${ }^{24}$. Prevalence of major depression was also significantly higher in those with HIV in the over 50 age group in rural South Africa ${ }^{47}$. Females, particularly those in the urban residency and those receiving a government grant, were affected ${ }^{47}$. Depression associated with HIV/AIDS has been linked with faster disease progression and reduced drug adherence $^{48}$. It has also been shown to be associated with less social support, worse quality of life, as well as higher HIV plasma viral loads, increased disease progression and higher mortality ${ }^{23,36,37,41,49,50}$. Suicidal ideation may be a cause for psychiatric referral in subjects who have been informed about a diagnosis of HIV/AIDS, and was found at 12.6\% and $10 \%$ prevalence in studies done in Lilongwe and rural Zambia ${ }^{24}$.

In patients with HIV, prevalence of mania is elevated though less so than depression ${ }^{21,25,34}$. Patients with mania secondary to HIV tend to present at a more advanced stage, are more likely to have developed AIDS, have a low CD4 count and have a higher prevalence of co-morbid dementia or cognitive slowing when compared to HIV-positive patients with primary mania ${ }^{20,23,26,34}$. The prevalence of mania in a population of HIV-positive patients was found to be similar to that of the general population, at $1.2-1.4 \%$, whereas the rate in patients with AIDS was $4.3-8 \%{ }^{20,26}$. Patients with mania secondary to HIV had more irritability, aggression, cognitive impairment and dementia and had higher rates of psychotic symptoms ${ }^{25}$. The frequency of affective episodes may increase following HIV infection in bipolar patients, and mania may occur having been given a diagnosis of $\mathrm{HIV}^{25}$.

The prevalence of generalized anxiety disorder, and adjustment disorder is increased in HIV-positive patients compared to HIV negative individuals ${ }^{26,50}$. These may arise from the distress associated with being diagnosed with the infection and from the implications of the diagnosis ${ }^{26,50}$.

Psychosis may occur as a direct result of HIV infection on the CNS, with the highest incidence reported among patients in later stages of HIV disease ${ }^{26}$. Cognitive dysfunction is also common among patients with HIV associated psychosis ${ }^{24,34,35}$. Cognitive impairment has also been found to be worse among HIV-positive individuals with psychosis in comparison to HIV negative individuals with psychosis ${ }^{21}$.

Dementia is commonly seen in patients with HIV, particularly when ART medication is not taken regularly ${ }^{51}$. Patel et al found a prevalence of HIV-associated dementia of $14.0 \%$ among HIV-positive adults ${ }^{51}$. Cognitive impairment in HIV has been associated with greatly increased mortality independent of other factors such as baseline clinical stage, CD4 count, serum haemoglobin concentration, ART use, and social and demographic characteristics ${ }^{52}$.

HIV infection has a number of psychiatric sequelae associated with it. Psychiatric illness is commonly the first presentation of HIV infection, as highlighted by a study in Uganda in which $43 \%$ of HIV-positive individuals did not have a prior episode of mental illness ${ }^{21}$. Several studies from high-income countries indicate a higher prevalence of major depressive disorder, milder depressive symptoms, anxiety disorder, ${ }^{53-55}$ mania, psychotic disorders, ${ }^{53,56-57}$ and substance abuse, ${ }^{58}$ as well as cognitive impairment ${ }^{56}$ among people infected with HIV. HIV infection may also exacerbate existing conditions such as Schizophrenia ${ }^{59}$.

\section{Adherence to Testing and Treatment}

It is thought that individuals with severe mental illness may lack competence to refuse testing or treatment for HIV. In a study in Thyolo, Malawi, $93.7 \%$ had the capacity to provide 
consent for HIV testing in outpatient settings, but other studies have found that only $80 \%-87 \%$ of patients with severe mental illness had adequate capacity to provide consent $t^{60}$. Comorbid diagnosis of mental disorder and HIV are associated with poorer ART adherence, faster disease progression and increased mortality ${ }^{61}$. Adhering to ART therapy requires, amongst other things, insight and capacity. Evidence from high-income countries shows that adherence may be negatively affected by depression, cognitive impairment, and alcohol and substance abuse, although adherence increases with close medical observation ${ }^{11}$. Poor adherence to ART may also be closely related to the central nervous system's adverse effects caused by these drugs, including Efavirenz, although this relationship is multi-factorial ${ }^{62}$. Adherence to ART is important in achieving viral suppression of HIV, thereby reducing infectiousness and helping prevent disease progression $^{63}$. Public health policies should therefore strive to maximize adherence ${ }^{50}$.

\section{Treatment of HIV}

The mainstay of HIV treatment is the so-called anti-retroviral therapy (ART) drugs. These drugs either interfere with the transcription of viral RNA into complimentary DNA (dependent on reverse transcriptase), or when viral proteins are produced and broken down into subunits via the protease enzyme $^{64}$. These drugs have significantly improved the clinical outcomes for patients with HIV. At the end of 2012, close to 10 million people were receiving ART in low and middle-income countries ${ }^{2}$. However, almost 19 million other people who were eligible for ART under new 2013 guidelines still did not have access to antiretroviral drugs ${ }^{2}$. Treatments consist of 'combination treatments,' which are classified as the nucleoside reverse transcriptase inhibitors, the nonnucleoside reverse transcriptase inhibitors and the protease inhibitors,${ }^{64}$ as well as the entry inhibitors, fusion inhibitors, and the integrase inhibitors ${ }^{65}$. These are exemplified as highly active antiretroviral therapy (HAART), which are a combination of protease and reverse transcriptase inhibitors. These aim to reduce viral loads and increase lymphocyte numbers, thus helping to decrease mortality and morbidity including HIV associated dementia and the incidence of opportunistic infections affecting the central nervous system (CNS) ${ }^{67}$.HIV infection has thus become a chronic disease with patients surviving for many years. It is important that one of these combination drugs crosses the blood brain barrier, yet this may paradoxically pre-dispose patients to neuropsychiatric side effects. Efavirenz is known to be one such agent that does cross the blood brain barrier ${ }^{68}$.

\section{Efavirenz}

Efavirenz is a medication commonly used as part of combination therapy for HIV treatment, and has been shown to be not inferior at reducing the viral load in HIV-positive patients compared to other preparations $s^{69,70}$. Hence, in Malawi, its use is now recommended as first line treatment, despite the noted side-effects. Current ART guidelines in Malawi recommend that those with symptoms of/or a history of mental illness should be commenced on the $6 \mathrm{~A}$ regimen, of which Efavirenz is not included ${ }^{71}$. These guidelines also advise that Nevirapine replace Efavirenz in those patients who experience severe side-effects such as psychosis $^{71}$. All those living with HIV should be initiated on ART irrespective of their CD4 count or clinical stage ${ }^{71}$.

Efavirenz is a selective non-nucleoside analog and non- competitive inhibitor of the reverse transcriptase enzyme of HIV. It attaches directly to this enzyme blocking DNA-RNA polymerase, causing the destruction of this catalytic site of the enzyme ${ }^{72}$. It is highly protein bound (around 99.7\%) to human plasma proteins, and is predominantly metabolised by the cytochrome P450 system. It has a prolonged half-life, permitting once daily dosing that has obvious benefits in terms of adherence and has good antiretroviral efficacy ${ }^{68}$.

\section{Neuropsychiatric side effects of Efavirenz}

Existing literature suggests a widely repeated association between antiretroviral drugs and psychiatric disorder, with Efavirenz being the most frequently cited. It may cause adverse psychiatric events ranging from $61-90 \%$ of patients, ${ }^{73-76}$ Reported side effects include: nightmares, headache, light-headedness, insomnia, confusion, lethargy, impaired concentration, amnesia, hallucinations, abnormal dreams, anxiety, de-personalisation, de-realisation, personality change, stream of thought troubles, mania, depression, suicidal thoughts, psychosis and hallucination ${ }^{74,76-87}$. In 2008, Marwaha found that African children and teenagers were experiencing hallucinogenic effects from smoking Efavirenz ${ }^{88}$.

Many of these adverse effects can be provoked by the virus itself, ${ }^{44-46}$ making it difficult to fully ascribe them to the effects of Efavirenz. Regardless of their origin, these side effects may lead to discontinuation of HAART therapy, but have been reported to resolve following discontinuation of Efavirenz and by adding appropriate psychiatric medication ${ }^{79}$. Spire et al found that female gender, unemployment, steady sexual partners and multiple episodes of depression were social, demographic and psychiatric risk factors for Efavirenz discontinuation ${ }^{89}$.

Some of these adverse effects may be associated with elevated serum levels of Efavirenz. Marzolini et al found that low levels predicted treatment failure and high levels were associated with CNS side effects ${ }^{90}$. The neurochemical mechanisms responsible for these adverse effects are not well understood. Side effects commonly appear within the first week of treatment, and some will disappear or diminish after several weeks ${ }^{11}$. Others may not diminish until after 200 days, ${ }^{92}$ whereas others may persist indefinitely ${ }^{78}$.

Several studies have highlighted the role of underlying psychiatric illness in predicting side effects to Efavirenz. Boly et al found that pre-existing depressive symptoms predicted neuropsychiatric side effects in HIV-1 positive patients treated with Efavirenz ${ }^{93}$ and Moreno et al suggested that pre-existing psychiatric diseases, such as Post Traumatic Stress Disorder (PTSD), could predispose to Efavirenz side effects $^{94}$. Others have indicated that young age and history of depression could be involved, ${ }^{95}$ as well as a history of chemical dependency. ${ }^{96}$

Allavena et al speculated that the combination of ART regimen may be of importance, since neuropsychiatric side effects were seen when switching from an Efavirenz ART regimen to an Efavirenz-Tenofovir combination ${ }^{97}$.

Of relevance, Efavirenz undergoes transformation by the CYP liver enzymes (mainly CYP2B6 and CYP3A4), and is thus subject to interactions with psychiatric medications ${ }^{88}$. For example, Fluoxetine and Fluvoxamine elevate the plasma level of Efavirenz and, thus, accentuate its effects on the $\mathrm{CNS}^{86}$. This is important in the Malawi context since Fluoxetine is one of the few commonly used anti-depressant 
medications. Also, genetic polymorphisms resulting in deficient alleles of the CYP2B6 isoform has been shown to be more prevalent in African populations ${ }^{98}$.

\section{Future Considerations}

As yet, there have been no case control studies undertaken in this population to establish more systematically which factors are associated with Efavirenz related psychiatric presentations. Little is known about what features are most common in neuropsychiatric presentation and what patient factors are associated with increased risk of developing these side effects or precipitating acute mental illness. One retrospective study by Von Giesen et al found no significant differences between Efavirenz and Nevirapine containing ART regimens with respect to memory, attention, psychomotor speed, CD4 count, and the manifestation of depression ${ }^{99}$. The study participants had similar demographic characteristics prior to therapy and a comparable outcome including adverse effects and neuropsychiatric symptoms. 99 It is therefore important to be able identify characteristics of neuropsychiatric presentations secondary to Efavirenz that distinguish this presentation from other forms of mental illness or characteristics of HIV infection itself; elucidating this will aid in the management of mental illnesses in those taking Efavirenz for co-morbid HIV infection. It would help determine those who may be at risk of this adverse reaction, and help to inform future HIV treatment strategies at local and national level, and contribute to the international evidence in this area.

Before initiating Efavirenz, patients should be screened for the presence of any mental disorder, or a history of one. Patients should be encouraged to attend follow-up clinics regularly, and clinic staff should be trained in recognizing neuropsychiatric side-effects. Follow-up should also be prolonged. The neuropsychiatric sequelae of Efavirenz should be treated according to severity, with intolerable ones warranting substitute therapy. This should consider interactions with psychotropic medications.

\section{References}

1.Robbins KE, Lemey P, Pybus OG et al. Human immunodeficiency virus type 1 epidemic: Date of origin, population history, and characterization of early strains. J. Virol. 2003;11:59-66.

2.Global Health Observatory data [internet] World Health Organization; c2016 [cited 2016 Dec 29]. Available from: http://www.who.int/gho/ hiv/en/

3.Malawi Demographic Health Survey 2010 [internet] The Demographic and Health Surveys Programme; c2010 [cited 2014 Mar 02]. Available from: https://dhsprogram.com/publications/publicationfr247-dhs-final-reports.cfm

4.Unaids.org [internet] UNAIDS; c2017 [cited 2017 Dec 15]. Available from: http://www.unaids.org/en/regionscountries/countries/malawi

5.Kauye F, Jenkins R, Rahman A. Training primary health care workers in mental health and its impact on diagnoses of common mental disorders in primary care of a developing country, Malawi: a clusterrandomized controlled trial. Psychol Med. 2014;44(3):657-666.

6.Wright J, Common S, Kauye F, Chiwandira C. Integrating community mental health within primary care in southern Malawi: A pilot educational intervention to enhance the role of health surveillance assistants. Int J Soc Psychiatry. 2014;60(2):155-161.

7.Stewart RC, Bunn J, Vokhiwa M, Umar E, Kauye F, Fitzgerald Met al. Common mental disorder and associated factors amongst women with young infants in rural Malawi. Soc Psychiatry Psychiatr Epidemiol. 2010;45(5):551-559.
8.Health Sector Strategic Plan 2011-2016 [internet] Government of Malawi; c2011 [cited 2014 Mar 02]. Available from: http://www.gov. scot/Topics/International/int-dev/mdp/healthsectorstrategicplan

9.Kauye F, Mafuta C. Country profile: Malawi. International Psychiatry. 2007; 4(1):9-11.

10. Proceedings of the 6th Annual Malawi Mental Health Research and Practice Development Conference; 2016 Mar 14-16; Blantyre, Malawi. [internet] c2016 [cited $2017 \mathrm{Feb}$ 03]. Available from: https://www. smmhep.org.uk/content/6th-annual-malawi-mental-health-researchand-practice-development-conference-2016

11. Prince M, Patel V, Saxena S et al. No health without mental health. Lancet. 2007;370(9590):859-877.

12.Campos MD, Podus D, Anglin MD, Warda U. Mental health need and substance abuse problem risk: acculturation among Latinas as a protective factor among CalWORKs applicants and recipients. J Ethn Subst Abuse. 2008;7(3):268-291.

13.Meade CS, Sikkema KJ. Psychiatric and psychosocial correlates of sexual risk behaviour among adults with severe mental illness. Community Ment Health J. 2007;43(2):153-169.

14.Angelino AF, Treisman GJ. Issues in co-morbid severe mental illnesses in HIV infected individuals. Int Rev Psychiatry. 2008;20(1):95101.

15.Collins PY, Holman AR, Freeman MC, Patel V. What is the relevance of mental health to HIV/AIDS care and treatment programs in developing countries? A systematic review. AIDS. 2006;20(12):15711582.

16.Kelly JA, Murphy DA, Sikkema KJ et al. Predictors of high and low levels of HIV risk behavior among adults with chronic mental illness. Psychiatr Serv. 1995;46(8):813-818.

17.Senn TE, Carey MP. HIV testing among individuals with a severe mental illness: review, suggestions for research, and clinical implications', Psychol. Med. 2009;39(3):355-363.

18.Bhatia R, Hartman C, Kallen MA, Graham J, Giordano TP. Persons newly diagnosed with HIV infection are at high risk for depression and poor linkage to care: results from the Steps Study. AIDS Behav. 2011;15(6):1161-70.

19.Venugopal D, Patil PB, Gupta D, Murali N, Kar N, Sharma PSV. Mania in HIV infection; Indian J Psychiatry. 2001;43(3):242-245.

20.Nakasujja N, Allebeck P, Agren H, Musisi S, Katabira E. Cognitive dysfunction among HIV positive and HIV negative patients with psychosis in Uganda. PLoS One. 2012;7(9):e44415. doi.org/10.1371/ journal.pone. 0044415

21.Nakasujja N, Skolasky RL, Musisi S et al. Depression symptoms and cognitive function among individuals with advanced HIV infection initiating HAART in Uganda. BMC Psychiatry. 2010;10(44). doi. org/10.1186/1471-244X-10-44.

22.Unnikrishnan B, Jagannath V, Ramapuram JT, Achappa B, Madi D. Study of depression and its associated factors among women living with HIV/AIDS in Coastal South India. ISRN AIDS. 2012;2012:684972. doi: $10.5402 / 2012 / 684972$

23.Alciati A, Fusi A, Monforte AD. New-onset delusions and hallucinations in patients infected with HIV. J Psychiatry Neurosci. 2001;26(3):229-234.

24.Mazenga A. Depression among adolescents at Baylor Clinic in Lilongwe. [dissertation]. St John of God College of Health sciences; 2009.

25. Owe-Larsson B, Säll L, Salamon E, Allgulander C. HIV infection and psychiatric Illness. Afr J Psychiatry. 2009;12(2):115-128.

26. Wainberg ML, McKinnon K, Elkington $\mathrm{K}$ et al. HIV risk behaviours among outpatients with severe mental illness in Rio de Janeiro, Brazil. World Psychiatry. 2008;7(3):166-172. 
27.Ferrando SJ, Chloe N. HIV-associated mania treated with electroconvulsive therapy and highly-active antiretroviral therapy. Psychosomatics. 2006; 47(2):170-174.

28.Senn TE, Carey MP. HIV Testing among individuals with a severe mental illness: review, suggestions for research, and clinical implications. Psychol Med. 2009;39(3):355-363.

29.Cruess DG, Evans DL, Repetto MJ, Gettes D, Douglas SD, Petitto JM. Prevalence, diagnosis, and pharmacological treatment of mood disorders in HIV disease. Biol Psychiatry. 2003;(54):307-316.

30.Cournos F, McKinnon K. HIV seroprevalence among people with severe mental illness in the United States: a critical review. Clin Psychol Rev. 1997;17(3):259-269.

31.Gottesman II, Groome CS. HIV/AIDS risks as a consequence of schizophrenia. Schizophr Bull. 1997;23(4):675-684.

32.Rosenberg SD, Goodman LA, Osher FC et al Prevalence of HIV, hepatitis B, and hepatitis $\mathrm{C}$ in people with severe mental illness. Am J Public Health. 2001;91(1):31-37.

33.Ayuso-Mateos JL, Montanes F, Lastra I, Pilcazo de la GJ, AyusoGutierrez JL. 'HIV infection in psychiatric patients: an unlinked anonymous study', Br J Psychiatry, 1997;170:181-185.

34.Wagner GJ, Goggin K, Remien RH. A closer look at depression and its relationship to HIV antiretroviral adherence,. Ann Behav Med. 2011;42(3):352-360.

35.Meade CS, Graff FS, Griffin ML, Weiss RD. HIV risk behaviour among patients with co-occurring bipolar and substance use disorders: associations with mania and drug abuse. Drug Alcohol Depend. 2008;92(1-3):296-300

36.Gonzalez-Torres MA, Salazar MA, Inchausti L et al. Lifetime sexual behaviour of psychiatric inpatients. Sex Med. 2010;7:3045-3056.

37.Chandra Carey MP, Carey KB, Rao P, Jairam KR, Thomas T. HIV risk behaviour among psychiatric inpatients: results from a hospital-wide screening study in Southern India. Int J STD AIDS 2003;14(8):532-538.

38.Reshma Gupta R, Dandu M, Packel L, et al. Depression and HIV in Botswana: a population-based study on gender-specific socioeconomic and behavioral correlates. PLoS ONE. 2010:5(12);e14252. doi. org/10.1371/journal.pone.0014252

39.Collins PY, Holman AR, Freeman MC, Patel V. What is the relevance of mental health to HIV/AIDS care and treatment programs in developing countries? A systematic review. AIDS. 2006;20(12):15711582.

40.Acuda SW, Sebit MB. Serostatus surveillance testing of HIV-I infection among Zimbabwean psychiatric inpatients, in Zimbabwe. Cent Afr J Med. 1996;42(9):254-257.

41.Lommerse K. HIV testing rate and seroprevalence among people attending a mental health clinic in rural Malawi [dissertation] University of Cape town; 2011.

42.Dube B, Benton T, Cruess DG, Evans DL. Neuropsychiatric manifestations of HIV infection and AIDS. J Psychiatry Neurosci. 2005;30(4):237-246.

43.Bell JE. The neuropathology of adult HIV infection. Rev Neurol. 1998; 154(12):816-29.

44.Neuenburg JK, Brodt HR, Herndier BG et al. HIV-related neuropathology, 1985 to 1999: rising prevalence of HIV encephalopathy in the era of highly active antiretroviral therapy. J Acquir Immune Defic Syndr. 2002;31(2):171-177.

45.Cournos F, McKinnon K, Sullivan G. Schizophrenia and comorbid human immunodeficiency virus or hepatitis C virus. J Clin Psychiatry. 2005;66(6):27-33.

46.Mwale CM. The prevalence of psychological distress and associated factors among people living with AIDS attending antiretroviral therapy clinics in Mzuzu, Malawi: a cross-sectional descriptive study [dissertation] University of Malawi; 2006.

47.Nyirenda M, Chatterji S, Rochat T, Mutevedzi P, Newell ML. Prevalence and correlates of depression among HIV-infected and affected older people in rural South Africa. J Affect Disord. 2013;151(1):31-8.

48.Marwick KF, Kaaya SF. Prevalence of depression and anxiety disorders in HIV-positive outpatients in rural Tanzania. AIDS Care. 2010;22(4):415-9.

49.Singh D, Berkman A, Bresnahan M. Seroprevalence and HIVassociated factors among adults with severe mental illness - a vulnerable population. S Afr Med J. 2009;99(7):523-527.

50.Joska J.A, Kaliski SZ, Benatar SR. Patients with severe mental illness: A new approach to testing for HIV. S Afr Med J. 2008;98:213217.

51.Patel VN, Mungwira RG, Tarumbiswa TF, Heikinheimo T, van Oosterhout JJ. High prevalence of suspected HIV-associated dementia in adult Malawian HIV patients. Int J STD AIDS. 2010;21(5):356-358.

52.Wilkie FL, Goodkin K, Eisdorfer C. Mild cognitive impairment and risk of mortality in HIV-1 infection. J Neuropsychiatry Clin Neurosci. 1998;10(2):125-32.

53.Owe-Larsson B, Sall L, Salamon E, Allgulander C. HIV infection and psychiatric illness. Afr J Psychiatry. 2009;12(2):115-128.

54.Starace F, Bartoli L, Aloisi MS et al. Cognitive and affective disorders associated to HIV infection in the HAART era: findings from the NeuroICONA study. Cognitive impairment and depression in HIV/AIDS. The NeuroICONA study. Acta Psychiatrica Scandinavica. 2002;106(1):20-26.

55.Ciesla JA, Roberts JE. Meta-analysis of the relationship between HIV infection and risk for depressive disorders. Am J Psychiatry. 2001;158:725-730.

56.Kieburtz K, Zettelmaier AE, Ketonen L, Tuite M, Caine ED. Manic syndrome in AIDS. Am J Psychiatry. 1991;148:1068-1070.

57. Sewell DD, Jeste DV, Atkinson JH, et al. HIV-associated psychosis: a study of 20 cases. San Diego HIV Neurobehavioural Reseach Centre Group. Am J Psychiatry. 1994;151:237-242.

58.Chander G, Himelhoch S, Moore RD. Substance abuse and psychiatric disorders in HIV positive patients: epidemiology and impact on antiretroviral therapy. Drugs. 2006;66(6):769-789.

59.Mauri MC, Fabiano L, Bravin S, Ricci C, Invernizzi G. Schizophrenic patients before and after HIV infection: as case-control study. Encephale. 1997;23:437-441.

60.Moser DJ, Schultz SK, Arndt S et al. Capacity to provide informed consent for participation in schizophrenia and HIV research. Am J Psychiatry. 2002;159:1201-1207.

61.Ickovics JR, Meade CS. Adherence to antiretroviral therapy among patients with HIV: a critical link between behavioral and biomedical sciences. J Acquir Immune Defic Syndr. 2002;31(3):S98-102.

62.Piacenti FJ. An update and review of antiretroviral therapy. Pharmacotherapy. 2006;26:1111-1133.

63.Lucas GM. Antiretroviral adherence, drug resistance, viral fitness and HIV disease progression: a tangled web is woven. J Antimicrob Chemother. 2005;55(4):413-416.

64.Araujo AAS, Alves IRA. Situacao atual e perspectivas da quimioterapia de combate ao HIV/AIDS. Terapeutica Anti-Retorviral. 2002; 8: 96-108.

65.Manning TG. Efavirenz and psychosis: Is there a link? Aust N Z J Psychiatry. 2012;46(7):687-688.

66. Gaida R, Truter I, Grobler C. Efavirenz: Areview of the epidemiology, severity and management of neuropsychiatric side-effects. S. Afr. J. Psyc. 2015;21(3):94-97. 
67.Sacktor N, Lyles RH, Skolasky R et al. HIV-associated neurologic disease incidence changes: Multicenter AIDS Cohort Study, 19901998. Neurology. 2001;56:257-260.

68. Cavalcante GIT, Capistrano Jr VLM, Cavalcante FSD et al. Implications of efavirenz for neuropsychiatry: a review. Int J Neurosci. 2010;120:739-745.

69.Gulick RM, Ribaudo HJ, Shikuma CM, et al. Triple-nucleoside regimens versus efavirenz-containing regimens for the initial treatment of HIV-1 infection. N Engl J Med. 2004;350(18):1850-1861.

70.Lennox JL, DeJesus E, Lazzarin A, et al. Safety and efficacy of raltegravir-based versus efavirenz-based combination therapy in treatment-naïve patients with HIV-1 infection: A multicentre, doubleblind, randomised controlled trial. Lancet. 2009;374(9692):796-806.

71.Hiv.health.gov.mw [Internet]. Ministry of Health: Department of HIV and AIDS. Government of Malawi; c2017 [cited 2017 Dec 15]. Available from: http://www.hiv.health.gov.mw

72.Clerq E. Antiviral drugs: Current state of the art. J Clin Virol. 2001;22:73-89

73.Blanch J, Martinez E, Rousaud A, et al. Preliminary data of a prospective study on neuro-psychiatric side effects after initiation of efavirenz. J. Acquir. Immune Defic. Syndr. 2001;27(4):336-343.

74.Jena A, Sachdeva RK, Sharma A, Wanchu A. Adverse drug reactions to nonnucleoside reverse transcriptase inhibitor-based antiretroviral regimen: A 24-week prospective study. J Int Assoc Physicians AIDS Care. 2009;8:318-322.

75.Perez-Molina JA. Safety and tolerance of efavirenz in different antiretroviral regimens: Results from a national multicenter prospective study in 1,033 HIV-infected patients. HIV Clin Trials. 2002;3:279-286.

76.Rihs TA, Begley K, Smith DE et al. Efavirenz and chronic neuropsychiatric symptoms: A cross-sectional case control study. HIV Med. 2006;7:544-548.

77.Gallego L, Barreiro P, Del Rio R, et al. Analyzing sleep abnormalities in HIV-infected patients treated with Efavirenz. Clin. Infect. Dis. 2004;38(3):430-432.

78.Fumaz CR, Munoz-Moreno JA, Molto J, et al. Long-term neuropsychiatric disorders on efavirenz-based approaches: quality of life, psychological issues, and adherence. J. Acquir. Immune Defic. Syndr. 2005;38(5):560-565.

79. Clifford DB, Evans S, Yang Y, et al. Impact of efavirenz on neuropsychological performance and symptoms in HIV-infected individuals. Ann Intern Med. 2005; 143(10):714-21.

80.Paterson DL, Swindells S, Brester M, Vergis EN. Adherence to protease inhibitor therapy and outcomes in patients with HIV infection. Ann Intern Med. 2000;133:21-30.

81.O'Mahony SM, Myint A, Steinbusch H, Leonard BE. Efavirenz induces depressive like behaviour, increased stress response and changes in the immune response in rats. Neuroimmunomodulation. 2005; 12:293-298.

82.Poulsen HD, Lublin HK. Efavirenz-induced psychosis leading to involuntary detention. AIDS. 2003;17:451-453.

83.Zalila H, Elloumi H, Gaha, et al. Troubles psychotiques aigus sous efavirenz chez un patient attaint de HIV. La Tunisie Medicale. 2010;88:119-121.
84.Foster R, Olajide D, Everall IP. Antiretroviral therapy-induced psychosis: case report and brief review of the literature. HIV Med. 2003;4:139-144.

85.De la Garza CL, Paoletti-Duarte S, Garcia-Martin C, GutierrezCasares JR. Efavirenz-induced psychosis. AIDS. 2001;15(14):19111912.

86.Arendt G, de Nocker D, von Giesen H-J, Nolting T. Neuropsychiatric side effects of efavirenz therapy. Expert Opin Drug Saf. 2007;6(2):147154.

87.Asselman V, Thienemann F, Pepper DJ et al. Central nervous system disorders after starting antiretroviral therapy in South Africa. AIDS. 2010;24:2871-2876.

88. Marwaha A. Getting High on HIV Drugs in S Africa [internet]. BBC News; 2008 [cited 2014 Mar 05]. Available from: http://news.bbc. co.uk/1/hi/world/africa/7768059.stm

89.Spire B, Carreiri P, Garzot MA, L'Henhaff M, Obadia Y. TRT 5 Group L Factors associated with efavirenz discontinuation in a large community based sample of patients. AIDS Care. 2004;16(5):558-564.

90.Marzolini C, Telenti A, Decosterd LA, Greub G, Buclin T. Efavirenz plasma levels can predict treatment failure and central nervous system side effects in HIV-1 infected patients. AIDS. 2001;15:71-75.

91.Lochet P, Peyriere H, Lotthe A, Mauboussin JM, Delmas B, Reynes J. Long-term assessment of neuro-psychiatric adverse reactions associated with efavirenz. HIV Med. 2003;4(1):62-66.

92.Hawkins T, Geist C, Young B et al. Comparison of neuropsychiatric side effects in an observational cohort of efavirenz and protease inhibitor-treated patients. HIV Clin Trials. 2005;6:187-196.

93.Boly L, Cafaro V, Dyner T. Depressive symptoms predict increased incidence of neuro-psychiatric side effects in patients treated with efavirenz. J Acquir Immune Defic Syndr. 2006;42(4):514-515.

94.Moreno A, Labelle C, Samet JH. Recurrence of post-traumatic stress disorder symptoms after initiation of antiretrovirals including efavirenz: a report of two cases. HIV Med. 2003;4(3):302-304.

95.Journot V, Chene G, De Castro N, et al. ALIZE STUDY GROUP: Use of efavirenz is not associated with a higher risk of depressive disorders: a substudy of the randomized clinical trial ALIZE-ANRS 099. Clin Infect Dis. 2006;42(12):1790-1799.

96.Peyriere H, Mauboussin JM, Roouanet I, Fabre J, Reynes J. Management of sudden psychiatric disorders related to efavirenz. AIDS. 2001;15:1323-1324.

97.Allavena C, Le Moal G, Michau C, Chiffoleau A, Raffi F. Neuropsychiatric adverse events after switching from an antiretroviral regimen containing efavirenz without tenofovir to an efavirenz regimen containing tenofovir: a report of nine cases. Antivir Ther. 2006;11(2):263-265.

98.Naidoo P, Chetty VV, Chetty M. Impact of CYP polymorphisms, ethnicity and sex differences in metabolism on dosing strategies: The case of efavirenz. Eur J Clin Pharmacol. 2014;70(4):379-389.

99. Von Giesen H-J, Koeller H, De Nocker D, Haslinger B, Arendt G. Long term safety and efficacy of NNRTI within the central nervous system. HIV Clin Trials. 2003;4(6):382-390. 